Recent guidelines published by the ACMG serve as a starting point to help laboratories and clinicians as they begin to navigate the use of genomic technologies in patient care. They will almost certainly be refined and revised, in response both to concerns by the clinical community and to emerging evidence. I think of the document as a rough draft that should now be discussed, debated, and improved.

In that spirit I address in the following editorial one of the many features of the new guidelines that has already sparked considerable discussion and controversy. Many more issues remain to be explored, and I hope that this journal can serve as a forum for ongoing, robust discussion of how to proceed as we attempt to harness new and powerful technologies to the benefit of our patients. The many questions that seem to be stirring controversy even at this early stage include the following:

- Should individuals be able to opt out of receiving incidental findings?

- Are such findings truly "incidental" given that some degree of intentional querying of a patient's genomic data is necessary to obtain them?

- How burdensome to laboratories, clinicians, and patients are such practices?

- How do the current recommendations square with notions of overt screening for disease?

I will address some of these issues in the future and hope to publish a diverse range of thoughtful opinions (and emergent data) from the genetics community pertaining to these subjects and others.

\title{
Return of results to the families of children in genomic sequencing: tallying risks and benefits
}

\author{
James P. Evans, MD, PhD ${ }^{1}$
}

It has long been the norm in our field that genetic testing of children for highly penetrant, adult-onset conditions is discouraged. ${ }^{1}$ After all, if a condition will not manifest for years, there is plenty of time for the child to decide, when he or she reaches the age of majority, whether to pursue such testing. Such a policy respects the right of children to make their own decisions when they will be prepared to do so, avoids the risk of psychological harm were their genetic status to be prematurely revealed, and, critically, poses little risk of harm to anyone.

We now find ourselves with a new set of recommendations ${ }^{2}$ from the American College of Medical Genetics and Genomics (ACMG) suggesting that families of children should be informed of specific genetic findings when they indicate a high likelihood of preventable, adult-onset disease.

What gives? Aren't these new guidelines in conflict with a seemingly long-settled issue as well as the College's previously stated position? Has the ACMG suddenly done an about-face regarding the return of results to children?

I don't think so. In fact, I think that both positions are consistent, reconcilable, and justified if we keep in mind that context matters greatly in clinical medicine. And the context of the two sets of recommendations couldn't be more different.
The new context (and the new set of recommendations) deals with the incidental discovery of a mutation in a child that confers a very high risk for a preventable, adult-onset disorder. This is a far cry from the situation in which a known familial risk exists, in which most agree that deliberate testing of a child offers little benefit.

In the traditional situation (around which the previous policies and discussion were contextualized), we face the question of whether to test a child for an adult-onset disease when there exists a known familial mutation or a high risk due to family history. In such a context there's little medical benefit to anyone in testing a child. At-risk adults can be readily tested should they desire, and the child can be tested when he or she is older and better able to weigh the pros and cons. Therefore, it continues to make perfect sense that children should be able to make a decision about testing when they reach adulthood because there are rarely competing interests that might compel us to overrule a child's autonomy.

However, in the setting of the incidental discovery of certain types of mutations, the context shifts radically-and here there is a far more compelling case for disclosure to the family. The incidental discovery of a mutation in a child that confers a high risk of an adult-onset condition has a direct impact on

${ }^{1}$ Department of Genetics, University of North Carolina, Chapel Hill, North Carolina, USA. Correspondence: James P. Evans (jpevans@med.unc.edu)

doi:10.1038/gim.2013.54 
the health of that child's parents; withholding such information has a high chance of causing overt harm.

Consider, for example, a child who undergoes whole-exome sequencing in an effort to arrive at a diagnosis for his dysmorphic features and developmental delay. Identification of a deleterious Lynch syndrome mutation represents potentially lifesaving information for the parents. Indeed, given the low incidence of new mutations in this syndrome, it is a near certainty that one of her parents is at very high risk for preventable cancer. Thus, unlike the context in which there already exists a known familial mutation (or an elevated risk due to family history) and where deliberately testing a child for the condition has few benefits, when the high risk is incidentally discovered there exists the distinct potential for near-term medical harm if that information is not divulged.

That isn't to say that communication of such incidentally discovered risks is entirely nonproblematic. After all, divulging the existence of the mutation may indeed violate a child's autonomy (although I would point out that even this violation isn't necessarily automatic-parents regularly make decisions not to tell their children a host of things). Violation of a child's autonomy should not be taken lightly and avoiding it mandates that the list of genes for which we consider such return be carefully considered and driven by evidence that disclosure is indeed beneficial. But when a high risk for a serious, preventable, adult-onset disease is uncovered, the countervailing harms that are likely to result from nondisclosure are substantial: the child's parents aren't given the surveillance they need to avoid a life-threatening condition. In this new incidental context, there is direct harm to others if certain, selected results are not returned. Moreover, there is the inarguable potential of overt harm to the child herself, in that having a parent diagnosed with preventable cancer stands to cause that child considerable harm.

So, keeping in mind the admonition we regularly offer to students that in genetics "the entire family is the patient," and assuming that we are seeking to minimize harm and maximize benefit to all in our practices, what's the tally of harms and benefits under these two policies? With regard to deliberately testing children for a known familial risk of adult-onset disease, there exists the risk of violating a child's autonomy and little in the way of countervailing harms if we simply wait until she is an adult and allow her to decide. This seems an easy call and is in concert with the long-standing decision by our community to not routinely test children for known familial risks in this context. The new recommendations address a different context and advise return of results with the incidental discovery of certain, selected mutations. In this new and different context, there is still the risk of harm that might result from violating a child's autonomy (if parents choose to divulge that information), but we now have dramatic benefits to both the parents (who stand a far smaller chance of disease and death if informed) and the child (who most likely would prefer that their parent not develop a life-threatening disease). My own view is that the balance in this situation is in favor of returning selected results as the new recommendations advise. It is difficult for me to envision how the withholding of such information to avoid possibly violating a child's autonomy warrants literally risking their own future and the life of their parent.

\section{DISCLOSURE}

The author declares no conflict of interest.

\section{REFERENCES}

1. Friedman Ross L, Salls HM, David KL, Anderson RR. Technical report: ethical and policy issues in genetic testing and screening of children. Genet Med 2013;15:234-245.

2. American College of Medical Genetics and Genomics. ACMG recommendations for reporting of incidental findings in clinical exome and genome sequencing. http://www.acmg.net/docs/ACMG_Releases_HighlyAnticipated_Recommendations_on_Incidental_Findings_in_Clinical_Exome_ and_Genome_Sequencing.pdf. 19 March 2013.

The views are those of the author and do not reflect official ACMG positions. 